\title{
ON THE BOUNDED SOLUTIONS OF A NONLINEAR CONVOLUTION EQUATION
}

\author{
Odo DiekManN \\ Mathematisch Centrum, $2^{\mathrm{e}}$ Boerhaavestraat 49, Amsterdam, Netherlands \\ and \\ HANS G. KAPER* \\ Applied Mathematics Division, Argonne National Laboratory, Argonne, IL 60439, U.S.A.
}

(Received 22 November 1977)

Key words: Nonlinear convolution equation on the real line, non-existence, asymptotic behaviour, uniqueness modulo translation, Tauberian methods, mathematical epidemiology.

\section{INTRODUCTION}

CERTAIN deterministic models for the spatial spread of an epidemic or an advantageous gene among a population along a line can be analyzed in terms of the nonlinear convolution equation

$$
u(x)=(g \circ u) * k(x) \quad x \in \mathbf{R},
$$

where $g \circ u$ is the composite of $g$ and $u, g \circ u(x)=g(u(x))$, and $*$ denotes convolution, $(\phi * \psi(x)=$ $\int_{\mathbf{R}} \phi(x-y) \psi(y) \mathrm{d} y$. In the epidemic model, $g$ typically has the form $g(x)=\alpha\left(1-\mathrm{e}^{-x}\right), \alpha$ some constant $(\alpha>1)$, cf. Diekmann [1], while in the genetic model $g$ is given by $g(x)=\left[\alpha x^{2}+\right.$ $\beta x(1-x)] /\left[\alpha x^{2}+2 \beta x(1-x)+\gamma(1-x)^{2}\right], \alpha, \beta$, and $\gamma$ positive constants, cf. Weinberger[2]. In both cases, $k$ is a nonnegative function, normalized such that $\int_{\mathbb{R}} k(x) \mathrm{d} x=1$.

We consider (1.1) under the following hypotheses:

$\left(\mathrm{H}_{g}^{\mathbf{1}}\right) \quad g: \mathbf{R}-\mathbf{R}$ is continuous; $g(0)=0$; there exists a $p>0$ such that $g(x)>x$ for $0<x<p$ and $g(p)=p$.

$\left(\mathbf{H}_{k}^{1}\right) \quad k: \mathbf{R} \rightarrow \mathbf{R}$ is nonnegative; $k \in L_{1}(\mathbf{R})$ with $\int_{\mathbf{R}} k(x) \mathrm{d} x=1 ; \int_{\mathbf{R}}|x| k(x) \mathrm{d} x<\infty$.

Further hypotheses on $g$ and $k$ will be introduced as needed.

Equation (1.1) has at least two constant solutions, viz., $u_{0}: x \mapsto 0$ and $u_{p}: x \mapsto p$. These we call trivial solutions. By a nontrivial solution of (1.1) we mean a Lebesgue measurable function $u: \mathbf{R} \rightarrow \mathbf{R}$ which satisfies (1.1) and the inequalities $0 \leqslant u(x) \leqslant p$ for almost all $x \in \mathbf{R}$ and which is not a trivial solution. We are interested in nontrivial solutions of (1.1) and in the solution of the corresponding inhomogeneous equation

$$
u(x)=(g \circ u) * k(x)+f(x) \quad x \in \mathbf{R},
$$

where $f$ is a given nonnegative function.

In Section 2 we establish various general properties of the solutions of (1.1). In sections 3 and 4 we prove some non-existence results, i.e., we establish conditions on $g$ and $k$ under which one can

\footnotetext{
* Work performed under the auspices of the U.S. Department of Energy.
} 
prove that the equation (1.1) does not have a nontrivial solution. These results are complementary to some earlier results of Weinberger [2] and of one of the authors [1] concerning the existence of nontrivial solutions of (1.1). As we will show, the existence or non-existence of such solutions hinges upon the distribution of the mass of the kernel $k$. Formally, this is determined by the existence or non-existence of real zeros of the function $\lambda \mapsto 1-g^{\prime}(0) K(\lambda)(\lambda \in \mathbb{R})$, where $K$ is the two-sided Laplace transform of $k$. The problem has some Tauberian aspects and part of our arguments are based on Pitt's form of Wiener's general Tauberian theorem. This approach is related to the use of Tauberian methods in the study of linear convolution equations by Karlin [3] and Essén [4], cf. [5, Chapter 9].

In Section 5 we study the inhomogeneous equation (1.2). In Section 6 we turn our attention to the case when a nontrivial solution of (1.1) exists and investigate the question of its uniqueness (modulo translation, as (1.1) is translation invariant). Here, our method of proof forces us to impose further conditions on $g$ and $k$.

In the final Section 7 we apply our results to a problem in mathematical epidemiology, viz., the travelling wave problem for the Kermack-McKendrick-Kendall model for the spatial spread of an epidemic, cf. [1,6-12].

Notation. $\mathbf{N}_{+}$denotes the set of all positive integers; $\mathbf{R}_{+}=\{x \in \mathbf{R}: x \geqslant 0\}$ and $\mathbf{R}_{-}=$ $\{x \in \mathbf{R}: x \leqslant 0\}$. The letter $C$ is used to denote a generic positive constant.

\section{PRELIMINARIES}

Starting from $k$ we form a sequence of functions $\left\{k^{n^{*}}: n \in \mathbf{N}_{+}\right\}$. We interpret $k^{1 *}$ as $k$, and define for $n \in \mathbf{N}_{+}, k^{(n+1)^{*}}:=k^{n *} * k$. Thus, $k^{n *} \in L_{1}(\mathbf{R})$ and $\int_{\mathbf{R}} k^{n *}(x) \mathrm{d} x=1$ for all $n$. Following Feller [13, Sections V.2, V.4], we call $x$ a point of increase of $k$ if $\int_{I} k(y) \mathrm{d} y>0$ for every open interval $I$ containing $x$, and we define $\sum:=\bigcup_{n=1}^{\infty} \sum_{n}$, where $\sum_{n}$ denotes the set formed by the points of increase of $k^{n *}$. We say that $k$ is concentrated on a set $I$ if $\int_{\mathbf{R} / I} k(x) \mathrm{d} x=0$. The following result will be used several times.

LEMMA 2.1. If $k$ is concentrated on $\mathbf{R}_{+}\left(\mathbf{R}_{-}\right)$, then there exists an $a \geqslant 0$ such that $\sum$ contains the interval $[a, \infty)((-\infty,-a])$. If $k$ is not concentrated on either $\mathbf{R}_{+}$or $\mathbf{R}_{-}$, then $\sum=\mathbf{R}$.

Proof. For an arbitrary real number $b>0$ we define $l(x):=\min \{k(x), b\}$. Then $l \in L_{\infty}(\mathbf{R}) \cap$ $L_{1}(\mathbf{R})$ and, furthermore,

$$
k^{2^{*}}(x) \geqslant l^{2}(x) \geqslant \int_{0}^{x} l(x-y) l(y) \mathrm{d} y=: L(x)
$$

We claim that $L(x)$ is uniformly continuous. Indeed, $\left|L\left(x_{1}\right)-L\left(x_{2}\right)\right| \leqslant b^{2}\left|x_{1}-x_{2}\right|+b \int_{\mathbf{R}} \mid l(y)-$ $l_{x_{1}-x_{2}}(y) \mid \mathrm{d} y$, where $l_{x}(y):=l(y-x)$, and the translation map $x \mapsto l_{x}$ from $\mathbf{R}$ into $L_{1}(\mathbf{R})$ is uniformly continuous, see [14, Section 1.1.5].

Suppose $\int_{\mathbf{R}_{+}} k(x) \mathrm{d} x>0$. Then $L$ cannot be identically zero on $\mathbf{R}_{+}$and, hence, there exist $x_{1} \quad 0$ and $\delta>0$ such that $k^{2^{*}}(x) \geqslant L(x)>0$ for $x \in\left(x_{1}, x_{1}+\delta\right)$. From this we deduce that $k^{4^{*}}(x)=\left(k^{2^{*}} * k^{2^{*}}\right)(x)>0$ for $x \in\left(2 x_{1}, 2 x_{1}+2 \delta\right)$ and, by induction, $k^{2 n^{*}}(x)>0$ for $x \in\left(n x_{1}, n x_{1}+\right.$ $n \delta), n \in \mathbf{N}_{+}$. For sufficiently large $n\left(n>\delta^{-1} x_{1}\right)$, successive intervals become overlapping and, 
consequently, $\sum$ contains an interval $[a, \infty)$ for some $a \geqslant 0$. A similar argument shows that if $\int_{\mathbf{R}_{-}} k(x) \mathrm{d} x>0$, then $\sum$ contains an interval $(-\infty,-a]$ for some $a \geqslant 0$.

Suppose now that both $\int_{\mathbf{R}_{+}} k(x) \mathrm{d} x>0$ and $\int_{\mathbf{R}_{-}} k(x) \mathrm{d} x>0$. Let $x_{0} \in \mathbf{R}$ be arbitrary. For sufficiently large $y$ we know that there exist positive integers $m$ and $n$ such that $k^{n *}(x)>0$ for $x$ in a neighbourhood of $\frac{1}{2} x_{0}+y$ and $k^{m^{*}}(x)>0$ for $x$ in a neighbourhood of $\frac{1}{2} x_{0}-y$. Consequently, $k^{(n+m)^{*}}(x)>0$ for $x$ in a neighbourhood of $x_{0}$ and $x_{0} \in \Sigma$.

The next lemma contains some basic information concerning nontrivial solutions of (1.1).

LEMMA 2.2. Any nontrivial solution $u$ of (1.1) is uniformly continuous, strictly positive, and such that $\inf \{u(x): x \in \mathbf{R}\}=0$.

Proof. Let $u$ be a nontrivial solution of (1.1). The uniform continuity of $u$ follows from the continuity of translation in $L_{1}(\mathbf{R})$ (cf. the proof of Lemma 2.1).

Next, we observe that $g \circ u(x) \geqslant u(x)$ for all $x \in \mathbf{R}$, so $u(x) \geqslant u * k(x)$ and, upon iteration, $u(x) \geqslant u * k^{n *}(x)$ for $n \in \mathbf{N}_{+}$. Hence, if $u\left(x_{0}\right)=0$ for some $x_{0} \in \mathbf{R}$, then $u * k^{n *}\left(x_{0}\right)=0$ for $n \in \mathbf{N}_{+}$, which implies $u(x)=0$ for all $x$ such that $x_{0}-x \in \sum$. On account of Lemma 2.1 this implies that $u(x)=0$ for all $x \in \mathbf{R}$ if $k$ is not concentrated on either $\mathbf{R}_{+}$or $\mathbf{R}_{-}$. If, on the other hand, $k$ is concentrated on a half-axis, $\mathbf{R}_{+}$say, then Lemma 2.1 implies that $u(x)=0$ for all $x \in\left(-\infty, x_{0}-a\right]$ for some $a \in \mathbf{R}_{+}$and, consequently, $u(x)=0$ for all $x \in \mathbf{R}$, since the equation (1.1) has to be satisfied. In all cases we thus arrive at the conclusion that $u(x)=0$ for all $x \in \mathbf{R}$, contrary to the assumption that $u$ is nontrivial.

Let $\alpha:=\inf \{u(x): x \in \mathbf{R}\}$ and suppose $\alpha>0$. Then $g \circ u(x) \geqslant \min \{g(y): \alpha \leqslant y \leqslant p\}$. Since $g$ is continuous, there exists a $\beta \in[\alpha, p]$ such that $g(\beta)=\min \{g(y): \alpha \leqslant y \leqslant p\}$. So $u(x)=(g \circ u) * k(x)$ $\geqslant g(\beta)=\alpha+(g(\beta)-\alpha) \geqslant \alpha+(g(\beta)-\beta)=\alpha+\varepsilon$, where $\varepsilon:=g(\beta)-\beta>0$. Hence, inf $\{u(x)$ : $x \in \mathbf{R}\} \geqslant \alpha+\varepsilon$, which is in contradiction with the definition of $\alpha$. We conclude that $\alpha=0$.

\section{THE FIRST NON-EXISTENCE RESULT}

In this section we give a non-existence result which can be proved by elementary arguments. Let $L$ be defined by

$$
L:=\limsup _{x \downarrow 0} \frac{g(x)}{x} .
$$

Note that $L \geqslant 1 ; L$ may be infinite. If $g$ is differentiable at the origin with derivative value $g^{\prime}(0)$, then $L=g^{\prime}(0)$.

LEMMA 3.1. Suppose $g$ is monotone nondecreasing on $[0, p]$. If $\int_{\mathbf{R}_{ \pm}} k(x) \mathrm{d} x>L^{-1}$, then any nontrivial solution $u$ of (1.1) is such that $\lim \inf u(x)>0$.

$$
x \rightarrow \pm \infty
$$

Proof. We prove the lemma only for the upper sign. Let $u$ be a nontrivial solution of (1.1) and suppose that $\lim \inf u(x)=0$. Choose a monotonically decreasing sequence $\left\{y_{n}: n \in \mathbf{N}_{+}\right\}$such that $y_{n} \downarrow 0$ and $g\left(y_{n}\right) / y_{n} \rightarrow L$ as $n \rightarrow \infty$. Define $x_{n}$ for $n=n_{0}, n_{0}+1, \ldots$, where $n_{0}$ is such that $u(0)>y_{n_{0}}$, by $x_{n}:=\sup \left\{x \in \mathbf{R}_{+}: u(y) \geqslant y_{n}\right.$ for all $\left.y \in[0, x]\right\}$. Then $u\left(x_{n}\right)=y_{n}$ and $x_{n} \rightarrow \infty$ as 
$n \rightarrow \infty$. Furthermore, as $g$ is monotone nondecreasing,

$$
u\left(x_{n}\right) \geqslant \int_{0}^{x_{n}} g \circ u\left(x_{n}-y\right) k(y) \mathrm{d} y \geqslant g \circ u\left(x_{n}\right) \int_{0}^{x_{n}} k(y) \mathrm{d} y,
$$

so $\left(g\left(y_{n}\right) / y_{n}\right) \int_{0}^{x_{n}} k(y) \mathrm{d} y \leqslant 1$ for all $n$. Letting $n \rightarrow \infty$ we obtain the inequality $L \int_{\mathbf{R}_{+}} k(y) \mathrm{d} y \leqslant 1$, a contradiction. We conclude that $\lim \inf u(x)>0$.

The following theorem is an immediate consequence of Lemmas 2.2 and 3.1.

THEOREM 3.2. Suppose $g$ is monotone nondecreasing on $[0, p]$. If $\int_{\mathbf{R}_{+}} k(x) \mathrm{d} x>L^{-1}$ and $\int_{\mathbf{R}_{-}} k(x) \mathrm{d} x>L^{-1}$, then there is no nontrivial solution of (1.1).

Proof. Let $u$ be a nontrivial solution of (1.1). Then either $\liminf _{x \rightarrow+\infty} u(x)=0$ or $\liminf _{x \rightarrow-\infty} u(x)=0$, by Lemma 2.2. However, from Lemma 3.1 we infer that $\liminf _{x \rightarrow+\infty} u(x)>0$ and $\liminf _{x \rightarrow-\infty} u(x)>0$, and we arrive at a contradiction. We conclude that there is no nontrivial solution of (1.1).

Remark 3.3. Up to this point we have not made use of the hypothesis that $\int_{\mathbf{R}}|x| k(x) \mathrm{d} x<\infty$, so all foregoing results remain valid if this hypothesis is actually violated.

\section{ANOTHER NON-EXISTENCE RESULT}

In the present section we establish a non-existence result whose proof is rather involved. It is based on various reformulations of (1.1) as an inhomogeneous linear convolution equation. First we rewrite (1.1) in the form

$$
u(x)-u * k(x)=\phi(x) \quad x \in \mathbf{R},
$$

where

$$
\phi(x):=(g \circ u-u) * k(x) .
$$

The function $\phi$ is nonnegative on $\mathbf{R}$ and we will exploit this fact in the investigation of solutions of (4.1), cf. Essén [4].

Let $m$ denote the first moment of the kernel $k$,

$$
m=\int_{\mathbf{R}} x k(x) \mathrm{d} x
$$

The first theorem requires barely more than a reference to the literature.

THEOREM 4.1. If $m=0$ then there is no nontrivial solution of (1.1).

Proof. It is known that the condition $m \neq 0$ is necessary and sufficient for the existence of a bounded continuous function $u$ such that $u(x)-u * k(x) \geqslant 0$, with strict inequality in some point, cf. [4, Theorem 3.1].

Having established this non-existence result for $m=0$, we may henceforth assume $m \neq 0$. 
Let

$$
n(x):=\left\{\begin{array}{l}
\int_{x}^{\infty} k(y) \mathrm{d} y \quad x \geqslant 0, \\
-\int_{-\infty}^{x} k(y) \mathrm{d} y \quad x<0 .
\end{array}\right.
$$

Jote that $n(x)=H(x)-\int_{-\infty}^{x} k(y) \mathrm{d} y$ and, consequently, $n^{\prime}=\delta-k$ in the sense of. distributions sere $H$ denotes the Heaviside step function and $\delta$ the Dirac distribution). The condition ${ }_{R}|x| k(x) \mathrm{d} x<\infty$ implies that $n \in L_{1}(\mathbf{R})$ with $\|n\|_{1}=\int_{\mathbb{R}}|x| k(x) \mathrm{d} x$,cf. [13, Section V.6]. The 'ourier transform $\hat{n}$ of $n$, which is a continuous function, is related to the Fourier transform of $k$ via the identity $\hat{n}(\lambda)=(1-\hat{k}(\lambda)) /(i \lambda)$ for $\lambda \neq 0$, while $\hat{n}(0)=m$. If $m \neq 0$, then $\hat{n}(\lambda) \neq 0$ for ll $\lambda \in \mathbf{R}$.

EMMA 4.2. Suppose $m>0$. If $u$ is any nontrivial solution of (1.1), then $\lim _{x \rightarrow-\infty} u(x)$ and $\lim _{x \rightarrow \infty} u(x)$ xist and $u(-\infty)=0, u(\infty)=p$.

Proof. Let $u$ be a nontrivial solution of (1.1). Then $u$ satisfies (4.1) with $\phi$ given by (4.2). We itegrate both members of (4.1) from $a$ to $x$,

$$
\int_{a}^{x} u(\xi) \mathrm{d} \xi-\int_{a}^{x} \int_{-\infty}^{\infty} u(\xi-\eta) k(\eta) \mathrm{d} \eta \mathrm{d} \xi=\int_{a}^{x} \phi(\xi) \mathrm{d} \xi .
$$

iterchanging the order of the integrals in the second term and integrating by parts we obtain ıe identity

$$
\int_{a}^{\infty} \int_{-\infty}^{\infty} u(\xi-\eta) k(\eta) \mathrm{d} \eta \mathrm{d} \xi=\int_{a}^{x} u(\xi) \mathrm{d} \xi-\int_{-\infty}^{\infty} n(x-\xi) u(\xi) \mathrm{d} \xi+\int_{-\infty}^{\infty} n(a-\xi) u(\xi) \mathrm{d} \xi
$$

here $n$ is defined by (4.4). Hence, $u$ satisfies

$$
u * n(x)-u * n(a)=\int_{a}^{x} \phi(\xi) \mathrm{d} \xi \quad x \in \mathbf{R} .
$$

nce $u$ is bounded and $n \in L_{1}(\mathbf{R}), u * n$ is bounded. Also, $\phi(x)$ is nonnegative for all $x \in \mathbf{R}$, so the tegral $\int_{a}^{x} \phi(\xi) d \xi$, besides being bounded, depends monotonically on $x$. It follows that $\lim$ $\phi(\xi) d \xi$ exists and hence, from (4.5), that $\lim u * n(x)$ exists. In fact,

$$
u * n(\infty)=u * n(a)+\int_{a}^{\infty} \phi(\xi) \mathrm{d} \xi
$$

ow, $n$ belongs to the Wiener class $W$ (i.e., $n \in L_{1}(\mathbf{R})$ and $\{\lambda \in \mathbf{R}: \hat{n}(\lambda)=0\}=\varnothing$ ), and $u$ is bounded Id uniformly continuous. It follows from Pitt's form of Wiener's fundamental Tauberian theorem, [5, Section 9.7] or [15, Section V.10], that $\lim _{x \rightarrow \infty} u(x)$ exists and is given by

$$
u(\infty)=m^{-1}\left(u * n(a)+\int_{a}^{\infty} \phi(x) \mathrm{d} x\right)
$$


Similarly, one shows that $\lim u(x)$ exists and is given by

$$
u(-\infty)=m^{-1}\left(n * n(a)-\int_{-\infty}^{a} \phi(x) \mathrm{dx}\right) .
$$

A simple argument shows that both $u(\infty)$ and $u(-\infty)$ satisfy the scalar equation $y=g(y)$, so $\{u(\infty), u(-\infty)\} \subset\{0, p\}$. Since $u(\infty)-u(-\infty)=\int_{-\infty}^{\infty} \phi(x) \mathrm{d} x>0$, we conclude that $u(-\infty)=0$ and $u(\infty)=p$.

Remark 4.3. The case $m<0$ can be reduced to the case $m>0$ by a change of variable $\tau: x \mapsto-x$.

The next step towards a non-existence result for $m \neq 0$ consists of an analysis of the rate of convergence of a nontrivial solution towards its limiting value 0 as $x \rightarrow-\infty$. The following lemma is needed in the proof of Lemma 4.5 .

Lemma 4.4. Let $f: \mathbf{R} \rightarrow \mathbb{R}$ be nonnegative, $f \in L_{1}(\mathbf{R})$. Define $f^{(0)}(x):=f(x)$ and, recursively, $f^{(k)}(x):=\int_{-\infty}^{x} f^{(k-1)}(y) \mathrm{d} y$ for those values of $k \in \mathbf{N}_{+}$for which $f^{(k-1)} \in L_{1}\left(\mathbb{R}_{-}\right)$. Then, for any $r \in \mathbb{R}$,

$$
\int_{-\infty}^{r}(r-x)^{k} f(x) \mathrm{d} x=k ! \int_{-\infty}^{r} f^{(k)}(x) \mathrm{d} x
$$

in the sense that if one side converges so does the other.

Proof. The identity given in the lemma follows upon induction from the identity

$$
\int_{-\infty}^{r}(r-x)^{k} f(x) \mathrm{d} x=k \int_{-\infty}^{r}(r-x)^{k-1} f^{(1)}(x) \mathrm{d} x .
$$

We omit the proof of the latter identity, as it is similar to the proof of Lemma 1 in $[13$, Section V.6].

Let $l$ be defined by

$$
l:=\liminf _{x \downarrow 0} \frac{g(x)}{x} .
$$

Note that $1 \leqslant l \leqslant L$, cf. (3.1); $l$ may be infinite. If $g$ is differentiable at the origin with derivative value $g^{\prime}(0)$, then $l=L=g^{\prime}(0)$.

LEMMA 4.5 Let $u$ be a nontrivial solution of (1.1) such that $\lim _{x \rightarrow-\infty} u(x)=0$. If $l>1$, then there exists a $\delta>0$ such that the integral $\int_{-\infty}^{\infty} \mathrm{e}^{-\lambda x} u(x) \mathrm{d} x$ converges for $\lambda \in S_{\delta}$, where $S_{\delta}:=\{\lambda \in \mathrm{C}: 0<$ $\operatorname{Re} \lambda<\delta\}$.

Proof. It suffices to prove the convergence of the integral $\int_{-\infty}^{r} \mathrm{e}^{-\lambda x} u(x) \mathrm{d} x$ for some $r<\infty$ and $\lambda$ real, $0<\lambda<\delta$.

Take $\alpha \in(1, l)$ and let $c$ be such that $\int_{-c}^{c} k(x) \mathrm{d} x=(\alpha+1) /(2 \alpha)$. Define a new function $k_{c}$ on $\mathbf{R}$ by putting $k_{c}(x):=k(x)$ if $|x| \leqslant c, k_{c}(x):=0$ if $|x|>c$. If $u$ is a nontrivial solution of (1.1), then $u(x)=(g \circ u)_{*} k_{c}(x)+\psi_{1}(x)$ for all $x \in \mathbf{R}$, with $\psi_{1}(x):=(g \circ u)_{*}\left(k-k_{c}\right)(x) \geqslant 0$. We rewrite the latter identity in the form

$$
u(x)-\frac{2 \alpha}{\alpha+1} u * k_{c}(x)=\frac{\alpha-1}{\alpha+1} u(x)+\psi(x) \quad x \in \mathbf{R}
$$


where

$$
\psi(x):=\frac{2}{\alpha+1}\left[\psi_{1}(x)+\psi_{2}(x)\right], \text { with } \psi_{2}(x):=(g \circ u-\alpha u) * k_{c}(x) .
$$

Let

$$
n_{c}(x):= \begin{cases}\frac{2 \alpha}{\alpha+1} \int_{x}^{\infty} k_{c}(y) \mathrm{d} y & x \geqslant 0, \\ -\frac{2 \alpha}{\alpha+1} \int_{-\infty}^{x} k_{c}(y) \mathrm{d} y & x<0 .\end{cases}
$$

Then supp $\left(n_{c}\right) \supset[-c, c]$. We integrate both members of (4.7) from $x$ to some $r<\infty$. Thus,

$$
\int_{x}^{r} u(\xi) \mathrm{d} \xi-\frac{2 \alpha}{\alpha+1} \int_{x}^{r} \int_{-\infty}^{\infty} u(\xi-\eta) k_{c}(\eta) \mathrm{d} \eta \mathrm{d} \xi=\frac{\alpha-1}{\alpha+1} \int_{x}^{r} u(\xi) \mathrm{d} \xi+\int_{x}^{r} \psi(\xi) \mathrm{d} \xi .
$$

Interchanging the order of the integrals in the second term and integrating by parts we obtain the identity

$$
u * n_{c}(r)-u * n_{c}(x)=\frac{\alpha-1}{\alpha+1} \int_{x}^{r} u(\xi) \mathrm{d} \xi+\int_{x}^{r} \psi(\xi) \mathrm{d} \xi,
$$

cf. (4.5). Because $\lim _{x \rightarrow-\infty} u(x)=0$, there exists $x_{\alpha} \in \mathbf{R}$ such that $g \circ u(x) \geqslant \alpha u(x)$ for all $x \in\left(-\infty, x_{\alpha}\right]$ and, hence, $\psi_{2}(x) \rightarrow 0$ for all $x \in\left(-\infty, x_{\alpha}-c\right]$. The same is then true of $\psi(x)$ and we conclude, as in the proof of Lemma 4.2, that $\lim _{x \rightarrow-\infty} \int_{x}^{r} u(\xi) \mathrm{d} \xi$ and $\lim _{x \rightarrow-\infty} \int_{x}^{r} \psi(\xi) \mathrm{d} \xi$ exist. Letting $x$ tend to $-\infty$ we obtain the identity

$$
\int_{-\infty}^{r} u(x) \mathrm{d} x=\frac{\alpha+1}{\alpha-1}\left[u * n_{c}(r)-\int_{-\infty}^{r} \psi(x) \mathrm{d} x\right] .
$$

If $r$ is chosen such that $r \leqslant x_{\alpha}-c$ it follows that

$$
\int_{-\infty}^{r} u(x) \mathrm{d} x \leqslant \frac{\alpha+1}{\alpha-1} \max \{u(x): x \in[r-c, r+c]\} \int_{\mathbf{R}}\left|n_{c}(x)\right| \mathrm{d} x .
$$

Next, we put $u^{(0)}(x):=u(x)$ and define $u^{(k)}(x):=\int_{-\infty}^{x} u^{(k-1)}(\xi) \mathrm{d} \xi, x \in \mathbf{R}$, for those values of $k \in \mathbf{N}_{+}$for which the integral exists. The functions $\psi^{(k)}$ are defined similarly. Integration of (4.7) from $-\infty$ to $x$ yields an equation for $u^{(1)}$ similar to (4.7), with $\psi$ replaced by $\psi^{(1)}$, from which one deduces the relation

$$
u^{(1)} * n_{c}(r)-u^{(1)} * n_{c}(x)=\frac{\alpha-1}{\alpha+1} \int_{x} u^{(1)}(\xi) \mathrm{d} \xi+\int_{x}^{r} \psi^{(1)}(\xi) \mathrm{d} \xi,
$$

cf. (4.8). The integrability of $u$ on $(-\infty, r)$ and the continuity of $u$ imply the boundedness of $u^{(1)}$ on $(-\infty, r+c]$. As before, it follows that $\lim _{x \rightarrow-\infty} \int_{x}^{r} u^{(1)}(\xi) \mathrm{d} \xi$ and $\lim _{x \rightarrow-\infty} \int_{x}^{r} \psi^{(1)}(\xi) \mathrm{d} \xi$ exist,

and, therefore,

$$
\int_{-\infty}^{r} u^{(1)}(x) \mathrm{d} x=\frac{\alpha+1}{\alpha-1}\left[u^{(1)} * n_{c}(r)-\int_{-\infty}^{r} \psi^{(1)}(x) \mathrm{dx}\right]
$$

$$
\int_{-\infty}^{r} u^{(1)}(x) \mathrm{d} x \leqslant \frac{\alpha+1}{\alpha-1} \max \left\{u^{(1)}(x): x \in[r-c, r+c]\right\} \int_{\mathbf{R}}\left|n_{c}(x)\right| \mathrm{d} x .
$$


Continuing in this manner we prove the existence of $u^{(k)}$ and obtain the sequence of inequalities

$$
\int_{-\infty}^{r} u^{(k)}(x) \mathrm{d} x \leqslant \frac{\alpha+1}{\alpha-1} \max \left\{u^{(k)}(x): x \in[r-c, r+c]\right\} \int_{\mathbf{R}}\left|n_{c}(x)\right| \mathrm{d} x,
$$

for $k \in \mathbf{N}_{+}$. Now, for each $k \in \mathbf{N}_{+}, u^{(k)}$ is a monotonically increasing function, so

$$
\begin{aligned}
\max & \left\{u^{(k)}(x): x \in[r-c, r+c]\right\}=u^{(k)}(r+c) \\
& \leqslant \int_{-\infty}^{r} u^{(k-1)}(x) d x+c u^{(k-1)}(r+c) \\
& \leqslant \delta^{-1} u^{(k-1)}(r+c),
\end{aligned}
$$

where $\delta:=\left[(\alpha+1)(\alpha-1)^{-1} \int_{\mathbf{R}}\left|n_{c}(x)\right| \mathrm{d} x+c\right]^{-1}$. Hence, by induction,

$$
\max \left\{u^{(k)}(x): x \in[r-c, r+c]\right\} \leqslant \delta^{-k} \max \{u(x): x \in[r-c, r+c]\} \leqslant p \delta^{-k} .
$$

Thus, there exists a positive constant $C$ such that

$$
\int_{-\infty}^{r} u^{(k)}(x) \mathrm{d} x \leqslant C \delta^{-k} \quad k \in \mathbf{N}_{+} .
$$

Next, we apply Lemma 4.4 to the function $u$ and conclude that each of the integrals $\int_{-\infty}^{r}(r-x)^{k} u(x) \mathrm{d} x$ converges. Moreover,

$$
\frac{1}{k !} \int_{-\infty}^{r}(r-x)^{k} u(x) \mathrm{d} x \leqslant C \delta^{-k}
$$

This result, in turn, implies the convergence of the infinite sum $\sum_{k=0}^{\infty}(k !)^{-1} \lambda^{k} \int_{-\infty}^{r}(r-x)^{k} u(x) d x$ and therefore of the integral $\int_{-\infty}^{r} e^{\lambda(r-x)} u(x) \mathrm{d} x$, at least for $\lambda<\delta$.

LemmA 4.6. Let $u$ be a nontrivial solution of (1.1). If the integral $\int_{-\infty}^{\infty} e^{-\lambda x} u(x) \mathrm{d} x$ converges in the strip $S_{\delta}$ (cf. Lemma 4.5) then so does the integral $\int_{-\infty}^{\infty} \mathrm{e}^{-\lambda x} k(x) \mathrm{d} x$.

Proof. It suffices to prove the convergence of the integral $\int_{-\infty}^{\infty} \mathrm{e}^{-\lambda x} k(x) \mathrm{d} x$ for $\lambda$ real, $0<\lambda<\delta$. Since $u$ is a nontrivial solution of (1.1) we have $u * k(x) \leqslant u(x)$ for all $x \in \mathbb{R}$, so $\int_{-\infty}^{\infty} \mathrm{e}^{-\lambda x} u * k(x) \mathrm{d} x \leqslant$ $\int_{-\infty}^{\infty} \mathrm{e}^{-\lambda x} u(x) \mathrm{d} x<\infty$ for any $\lambda \in(0, \delta)$. On the other hand, $\int_{-\infty}^{\infty} \mathrm{e}^{-\lambda x} u * k(x) \mathrm{d} x=\left(\int_{-\infty}^{\infty} \mathrm{e}^{-\lambda x} k(x) \mathrm{d} x\right)$ $\left(\int_{-\infty}^{\infty} \mathrm{e}^{-\lambda x} u(x) \mathrm{d} x\right)$, in the sense that if the integral on the left hand side exists then so do the integrals on the right hand side. Hence, $\int_{-\infty}^{\infty} \mathrm{e}^{-\lambda x} k(x) \mathrm{d} x$ exists for $\lambda \in(0, \delta)$ and satisfies the inequality $\int_{-\infty}^{\infty} \mathrm{e}^{-\lambda x} k(x) \mathrm{d} x \leqslant 1$ there.

We remark that Lemmas 4.5 and 4.6 together imply that, if $l>1$ then a necessary condition for the existence of nontrivial solutions of (1.1) is that $\int_{-\infty}^{\infty} \mathrm{e}^{-\lambda x} k(x) \mathrm{d} x$ converges in $S_{\delta}$ for some $\delta>0$.

The above results lead us to define a real number $\Lambda_{k}$,

$$
\Lambda_{k}:=\sup \left\{\lambda \in \mathbf{R}: \int_{\mathbf{R}} \mathrm{e}^{-\lambda x} k(x) \mathrm{d} x<\infty\right\}
$$

and to associate with each nontrivial solution $u$ of (1.1) a number $\Lambda_{u}$ thus,

$$
\Lambda_{u}:=\sup \left\{\lambda \in \mathbb{R}: \int_{\mathbb{R}} \mathrm{e}^{-\lambda x} u(x) \mathrm{d} x<\infty\right\} .
$$


If follows from Lemma 4.6 that $\Lambda_{u} \leqslant \Lambda_{k}$. Furthermore, let

$$
\begin{aligned}
& K(\lambda):=\hat{k}(-i \lambda)=\int_{\mathbf{R}} \mathrm{e}^{-\lambda x} k(x) \mathrm{d} x, \\
& U(\lambda):=\hat{u}(-i \lambda)=\int_{\mathbf{R}} \mathrm{e}^{-\lambda x} u(x) \mathrm{d} x,
\end{aligned}
$$

whenever the defining integrals converge. It is a standard result from Laplace transform theory that if $\Lambda_{u}>0$ then $U$ is analytic in the strip $S_{u}:=\left\{\lambda \in \mathbf{C}: 0<\operatorname{Re} \lambda<\Lambda_{u}\right\}$, cf. [15, Section VI.4]. The following result is a consequence of the positivity of $u(x)$.

LEMMA 4.7. If $u$ is a nontrivial solution of (1.1) and $0<\Lambda_{u}<\infty$, then $U(\lambda)$ is singular at $\lambda=\Lambda_{u}$.

\section{Proof. Cf. [15, Section II.5].}

At this point we make two further hypotheses concerning the functions $g$ and $k$ :

$\left(H_{g}^{2}\right)$ the function $g$ is differentiable at 0 with derivative value $g^{\prime}(0)$ and $g(x)=g^{\prime}(0) x+O\left(x^{1+\varepsilon}\right)$ as $x \downarrow 0$ for some $\varepsilon>0$;

$\left(H_{k}^{2}\right) \quad$ the kernel $k$ is exponentially small at $-\infty$, i.e., there exists a $\delta>0$ such that $\mathrm{e}^{-\lambda x} k(x)$ is bounded for $\lambda \in(0, \delta)$.

Under these additional hypotheses we can prove the following result.

LEMMA 4.8. Let $u$ be a nontrivial solution of (1.1) with $\lim _{x \rightarrow-\infty} u(x)=0$. If $0<\Lambda_{u}<\Lambda_{k}$, then $1-g^{\prime}(0) K\left(\Lambda_{u}\right)=0$.

Proof. We rewrite (1.1) as a linear inhomogeneous integral equation,

$$
u(x)-g^{\prime}(0) u * k(x)=r(x) \quad x \in \mathbf{R},
$$

where $r(x):=\left(g \circ u-g^{\prime}(0) u\right) * k(x)$. A two-sided Laplace transform yields the equation

$$
\left(1-g^{\prime}(0) K(\lambda)\right) U(\lambda)=R(\lambda)
$$

which is valid for all $\lambda \in S_{u}$. In fact, the right abscissa of convergence of $R$ lies to the right of $\Lambda_{u}$. This can be shown in the following manner. Because of the hypothesis $\left(H_{k}^{2}\right),\left|u(x) \mathrm{e}^{-\lambda x}\right|=$ $\left|(g \circ u) * k(x) \mathrm{e}^{-\lambda x}\right| \leqslant C \int_{\mathbf{R}} g \circ u(y) \mathrm{e}^{-y \operatorname{Re} \lambda} \mathrm{d} y$. The integral is bounded for $\operatorname{Re} \lambda$ sufficiently small, since $g(x)<C x$ for $x$ near zero $(x>0)$. Hence, using the hypothesis $\left(\mathrm{H}_{g}^{2}\right)$ we obtain for $\lambda \in\left(0, \Lambda_{\nu}\right)$ and $v$ sufficiently small $(v>0)$,

$$
\begin{aligned}
& \left|\int_{-\infty}^{\infty} \mathrm{e}^{-(\lambda+v) x}\left(g \circ u-g^{\prime}(0) u\right) * k(x) \mathrm{d} x\right| \\
= & K(\lambda+v) \int_{-\infty}^{\infty} \mathrm{e}^{-(\lambda+v) x}\left|\left(g \circ u-g^{\prime}(0) u\right)(x)\right| \mathrm{d} x \\
\leqslant & C K(\lambda+v) \int_{-\infty}^{\infty} \mathrm{e}^{-\lambda x} u(x)\left(\mathrm{e}^{-(v / \varepsilon) x} u(x)\right)^{\varepsilon} \mathrm{d} x
\end{aligned}
$$




$$
\leqslant C K(\lambda+v) U(\lambda)\left(\sup \left\{\mathrm{e}^{-(v / \varepsilon) x} u(x): x \in \mathbf{R}\right\}\right)^{\varepsilon} .
$$

This result shows that the abscissa of convergence of $R$ lies to the right of $\Lambda_{u}$. Consequently, $R(\lambda)$ is analytic in the neighborhood of $\lambda=\Lambda_{u}$. Since $U(\lambda)$ is singular at $\lambda=\Lambda_{u}$ (Lemma 4.7) we conclude from (4.11) that $1-g^{\prime}(0) K(\lambda)=0$ at $\lambda=\Lambda_{u}$.

The preceding lemmas lead to the following non-existence theorem.

TheOREM 4.9. Suppose $m>0, g^{\prime}(0)>1$ and $1-g^{\prime}(0) K(\lambda) \neq 0$ for all $\lambda \in\left[0, \Lambda_{k}\right)$. If there exists a $\lambda<\Lambda_{k}$ such that $\int_{-\infty}^{0} \mathrm{e}^{-\lambda x} k(x) \mathrm{d} x>\left(g^{\prime}(0)\right)^{-1}$, then there is no nontrivial solution of (1.1).

Proof. Suppose (1.1) has a nontrivial solution $u$. Then $\lim _{x \rightarrow-\infty} u(x)=0$ by Lemma 4.2, and $\Lambda_{u}>0$ by Lemma 4.5 and the definition (4.10) of $\Lambda_{u}$. For any $\alpha \in\left(1, g^{\prime}(0)\right)$ there exists an $x_{\alpha}$ such that $g \circ u(x) \geqslant \alpha u(x)$ for all $x \leqslant x_{\alpha}$. Take any $c>0$. Then $u(x) \geqslant \alpha \int_{-c}^{\infty} u(x-y) k(y) \mathrm{d} y$ whenever $x \leqslant x_{\alpha}-c$ and, consequently,

$$
u(x) \mathrm{e}^{-\lambda x} \geqslant \alpha \int_{-c}^{\infty} \mathrm{e}^{-\lambda(x-y)} u(x-y) \mathrm{e}^{-\lambda y} k(y) \mathrm{d} y
$$

for real $\lambda$. Now, $\lim \inf u(x) \mathrm{e}^{-\lambda x}=0$ for any $\lambda \in\left[0, \Lambda_{u}\right)$, so if we take a monotonically decreasing sequence $\left\{y_{n}: n \in \mathbf{N}_{+}\right\}$such that $y_{n} \downarrow 0$ as $n \rightarrow \infty$, and define $x_{n}$ for $n=n_{0}, n_{0}+1, \ldots$, where $n_{0}$ is such that $u(0)>y_{n_{0}}$, by $x_{n}:=\inf \left\{x \in \mathbf{R}_{-}: u(y) \mathrm{e}^{-\lambda y} \geqslant y_{n}\right.$ for all $\left.y \in[x, 0]\right\}$, then $u\left(x_{n}\right) \mathrm{e}^{-\lambda x_{n}}=y_{n}$ and $x_{n} \rightarrow-\infty$ as $n \rightarrow \infty$. Now, $[-c, 0] \subset\left[x_{n}, 0\right]$ for $n$ sufficiently large, so $u\left(x_{n}-y\right) e^{-\lambda\left(x_{n}-y\right)} \geqslant y_{n}$ for all $y \in[-c, 0]$ and

$$
\begin{aligned}
y_{n}=u\left(x_{n}\right) \mathrm{e}^{-\lambda x_{n}} & \geqslant \alpha \int_{-c}^{\infty} \mathrm{e}^{-\lambda\left(x_{n}-y\right)} u\left(x_{n}-y\right) \mathrm{e}^{-\lambda y} k(y) \mathrm{d} y \\
& \geqslant \alpha \int_{-c}^{0} \mathrm{e}^{-\lambda\left(x_{n}-y\right)} u\left(x_{n}-y\right) \mathrm{e}^{-\lambda y} k(y) \mathrm{d} y \\
& \geqslant \alpha y_{n} \int_{-c}^{0} \mathrm{e}^{-\lambda y} k(y) \mathrm{d} y .
\end{aligned}
$$

Thus, we obtain the inequality

$$
\alpha \int_{-c}^{0} \mathrm{e}^{-\lambda y} k(y) \mathrm{d} y \leqslant 1
$$

which is valid for any $\lambda \in\left[0, \Lambda_{u}\right)$. If $\Lambda_{u}$ were strictly less than $\Lambda_{k}$, then the function $\lambda \mapsto 1-g^{\prime}(0) K(\lambda)$ would have a zero at $\Lambda_{u}$ by Lemma 4.8 , i.e., inside the interval $\left[0, \Lambda_{k}\right)$, contrary to the hypothesis of the theorem. Hence, $\Lambda_{u}=\Lambda_{k}$ and the inequality (4.12) holds for all $\lambda \in\left[0, \Lambda_{k}\right)$. Since $\alpha$ and $c$ were chosen arbitrarily subject to the constraints $1<\alpha<g^{\prime}(0)$ and $c>0$ we conclude that the assumption of the existence of a nontrivial solution of (1.1) leads to the validity of the inequality $g^{\prime}(0) \int_{-\infty}^{0} \mathrm{e}^{-\lambda y} k(y) \mathrm{d} y \leqslant 1$ for all $\lambda \in\left[0, \Lambda_{k}\right)$. This proves the theorem.

If $\Lambda_{k}=\infty$, or if $K(\lambda) \rightarrow \infty$ for $\lambda \uparrow \Lambda_{k}$, then there certainly exists a $\lambda<\Lambda_{k}$ such that $\int_{-\infty}^{0} \mathrm{e}^{-\lambda x} k(x) \mathrm{d} x>\left(g^{\prime}(0)\right)^{-1}$. If, on the other hand, $K(\lambda)$ approaches a finite limit as $\lambda \uparrow \Lambda_{k}$, then 
Theorem 4.9 is not necessarily applicable. In that case, one can conclude from a Tauberian theorem for Laplace transforms, cf. [15, Section V.4], that the function $u_{\lambda}: \mathrm{x} \mapsto u(x) \mathrm{e}^{-\lambda x}$ belongs to $L_{1}(\mathbf{R})$ for $\lambda=\Lambda_{k}$. This implies that, under the hypothesis that $k_{\lambda}: x \mapsto k(x) \mathrm{e}^{-\lambda x}$ is bounded for $\lambda=\Lambda_{k}, u_{\lambda}$ is bounded for $\lambda=\Lambda_{k}$. Then one can repeat the analysis of the present section, starting from the identity $u_{\lambda}(x)=\int_{\mathbb{R}} \mathrm{e}^{-\lambda y}(g \circ u)(y) k_{\lambda}(x-y) \mathrm{d} y$ for $\lambda=\Lambda_{k}$, to obtain a non-existence result. We do not elaborate this idea any further.

Remark 4.10. The condition that $k$ has a finite first absolute moment $\left(\int_{\mathbf{R}}|x| k(x) \mathrm{d} x<\infty\right)$ has been used only once, in Lemma 4.2 to prove that any nontrivial solution of (1.1) has limits as $x \rightarrow \infty \pm$. The proofs of Lemmas 4.5 through 4.8 and Theorem 4.9 proceeded without an explicit reference to this condition. It remains an open question whether, if $k$ does not have finite first absolute moment, there exist nontrivial solutions of (1.1) which do not have limits as $x \rightarrow \pm \infty$.

\section{THE INHOMOGENEOUS EQUATION}

As the greater part of the proofs in the foregoing section were based on inequalities, rather than equalities, the same or similar arguments can be used in the analysis of the inhomogeneous equation

$$
u(x)=(g \circ u) * k(x)+f(x) \quad x \in \mathbf{R} .
$$

In fact, let $f$ satisfy the following hypothesis:

$\left(H_{f}\right) \quad f: \mathbf{R} \rightarrow \mathbf{R}$ is uniformly continuous, nonnegative and not equal to the zero function.

We then have the following result.

THeOREM 5.1. Suppose $g(x) \geqslant p$ for $x \geqslant p$. The same hypotheses on $g$ and $k$ which lead to the nonexistence results of Theorem 4.1 and Theorem 4.9 guarantee that any bounded nonnegative solution $u$ of (5.1) necessarily satisfies the inequality $u(x) \geqslant p$ for all $x \in \mathbf{R}$.

Proof. Let $u$ satisfy (5.1). We introduce the function $w$ by the definition $w(x):=\min \{u(x), p\}$, $x \in \mathbf{R}$. Then $g \circ u(x) \geqslant w(x)$ for all $x \in \mathbf{R}$, so $u(x) \geqslant(g \circ u) * k(x) \geqslant w * k(x)$. But $w(x) \leqslant p$ for all $x \in \mathbf{R}$, so $w * k(x) \leqslant p \int_{\mathbf{R}} k(x) \mathrm{d} x=p$. Combining these two results we see that $w$ satisfies the inequality $w * k(x) \leqslant \min \{u(x), p\}=w(x)$ for all $x \in \mathbf{R}$. Under the hypotheses of Theorem 4.1, this inequality leads to the conclusion that $w$ is constant, $w(x)=c$ say. The case $0 \leqslant c<p$ is excluded, because then $u(x)=c$ and (5.1) cannot be satisfied. Hence, $w(x)=p$ and $u(x) \geqslant p$ for all $x \in \mathbf{R}$. Under the hypotheses of Theorem 4.9 it follows that $w(-\infty):=\lim w(x)$ and $w(\infty):=\lim w(x)$

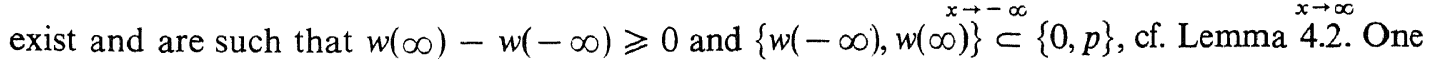
shows as in Lemma 2.2 that $w(x)>0$ for all $x \in \mathbf{R}$, and that either $w(x)=p$ for all $x \in \mathbf{R}$ or $\inf \{w(x): x \in \mathbf{R}\}=0$. If follows that either $w(x)=p$ for all $x \in \mathbf{R}$ or $w(-\infty)=0$. In the latter case, the chain of arguments leading from Lemma 4.4 to Theorem 4.9 can be repeated mutatis mutandis. Under the conditions of Theorem 4.9 the assumption that there exists a nontrivial $w$ such that $\lim w(x)=0$ leads to a contradiction, so necessarily $w(x)=p$, i.e. $u(x) \geqslant p$, for all $x \in \mathbf{R}$. 
Remark 5.2. A close examination of the proof of Theorem 5.1 shows that the condition $\left(\mathrm{H}_{f}\right)$ can be weakened somewhat if there exists a monotonically nondecreasing function $g_{0}$ on $[0, p]$ such that $g(x) \geqslant g_{0}(x)>x$ for all $x \in(0, p)$.In fact, let $f: \mathbf{R} \rightarrow \mathbf{R}$ be such that $f(x) \geqslant f_{0}(x)$ for all $x \in \mathbf{R}$, where $f_{0} . \mathbf{R} \rightarrow \mathbf{R}$ satisfies $\left(\mathrm{H}_{f}\right)$. Suppose $u$ is a solution of (5.1). Consider the equation

$$
v(x)=\left(g_{0} \circ v\right) * k(x)+f_{0}(x) \quad x \in \mathbb{R} .
$$

The minimal solution of (5.2) can be constructed via the method of successive iterations, $v^{(0)}(x):=f_{0}(x), v^{(n)}(x):=\left(g_{0} \circ v^{(n-1)}\right) * k(x)+f_{0}(x) \quad\left(n \in \mathbf{N}_{+}\right)$. Any of these iterates is majorized by the solution $u$ of (5.1). The monotonicity of $g_{0}$ implies that the sequence $\left\{v^{(n)}(x): n \in \mathbf{N}_{+}\right\}$is monotonically nondecreasing for each $x \in \mathbb{R}$, so the sequence converges to a limit function, $v$ say, which satisfies the equation (5.2) and the inequality $v(x) \leqslant u(x)$ for all $x \in \mathbf{R}$. Suppose now that the function $g_{0}$ is such that any bounded nonnegative solution $v$ of (5.2) necessarily satisfies the inequality $v(x) \geqslant p$ for all $x$, according to the theorem, then the solution $u$ of (5.1) must also satisfy the inequality $u(x) \quad p$ for all $x \in \mathbb{R}$.

\section{ASYMPTOTIC BEHAVIOUR AND UNIQUENESS CRITERIA}

The key assumption leading to the non-existence result of Theorem 4.9 is that the function $\lambda \mapsto 1-g^{\prime}(0) K(\lambda)$ does not vanish in the interval $\left[0, \Lambda_{k}\right)$. In the present section we will drop this assumption, i.e., we assume that the function $\lambda \mapsto 1-g^{\prime}(0) K(\lambda)$ has a real positive zero. The nonnegativity of $k$ implies that $K$ is a convex function on $\left[0, \Lambda_{k}\right)$, so $\lambda \mapsto 1-g^{\prime}(0) K(\lambda)$ has at most two real positive zeros. Let $\sigma$ denote the smallest positive zero; we assume that it is simple.

In Section 4 we established various conditions which guarantee that any nontrivial solution of (1.1) has the property that $\lim u(x)=0$. We also analyzed the rate of convergence of $u(x)$ to its limiting value 0 in terms of the Laplace transform of $u$. Our first objective in the present section is to establish conditions which guarantee that any nontrivial solution of (1.1) has the property that $u(x) \sim \mathrm{e}^{\sigma x}$ as $x \rightarrow-\infty$. We will then use this knowledge of the asymptotic behaviour to establish a uniqueness result for the nontrivial solutions of (1.1).

The following lemma is a modified version of Ikehara's Theorem and will be needed in the proof of Theorem 6.2 .

LEMMA 6.1. If the real-valued function $\phi$ is nonnegative and nonincreasing on $\mathbf{R}_{+}$, and there exists a $\tau>0$ such that the integral $f(\lambda)=\int_{0}^{\infty} \mathrm{e}^{-\lambda x} \phi(x) \mathrm{d} x$ converges for $\operatorname{Re} \lambda>-\tau$, and if, furthermore, for some constant $A$ and some function $h: \mathbf{R} \rightarrow \mathbf{R}$,

$$
\lim _{x \downarrow-\tau}\left[f(x+i y)-A(\tau+x+i y)^{-1}\right]=h(y),
$$

uniformly on compact subsets of $\mathbf{R}$, then

$$
\lim _{x \rightarrow \infty} \phi(x) \mathrm{e}^{\tau x}=A .
$$

Proof. Analogous to the proof of Ikehara's Theorem, see e.g. [15, Section V.17].

TheOREM 6.2. Suppose $m>0, g^{\prime}(0)>1$ and $g(x) \leqslant g^{\prime}(0) x$ for $x \in[0, p]$. If $u$ is a monotone nondecreasing nontrivial solution of (1.1), then there exists a positive constant $A$ such that $\lim _{x \rightarrow-\infty} u(x) \mathrm{e}^{-\sigma x}=A$. 
Proof. Let $u$ be a monotone nondecreasing nontrivial solution of (1.1). It follows from Lemma 4.5 and the definition (4.10) of $\Lambda_{u}$ that $U(\lambda)$ is defined for $\lambda \in\left[0, \Lambda_{u}\right)$ for some $\Lambda_{u}>0$. Moreover, $\left[1-g^{\prime}(0) K(\lambda)\right] U(\lambda)=R(\lambda)$, cf. (4.11). The continuity of $g$, together with the properties $g(0)=0$, $g^{\prime}(0)>1$, and $g(p)=p$, imply that the inequality $\leqslant$ in the condition $g(x) \leqslant g^{\prime}(0) x$ for $x \in[0$. $p]$ in the statement of the theorem, is a strict inequality, $g(x)<g^{\prime}(0) x$, at least on some subinterval of $[0, p]$. Hence, $g \circ u(x)<g^{\prime}(0) u(x)$ on a set of positive measure on $\mathbf{R}$ and, consequently, the function $R: \lambda \mapsto \int_{\mathbb{R}} \mathrm{e}^{-\lambda x}\left(g \circ u-g^{\prime}(0) u\right) * k(x) \mathrm{d} x$ does not vanish at $\lambda=\sigma$. Thus,

$$
U(\lambda)=\frac{R(\lambda)}{1-g^{\prime}(0) K(\lambda)} \sim \frac{R(\sigma)}{g^{\prime}(0) K^{\prime}(\sigma)}(\sigma-\lambda)^{-1} \quad \text { as } \lambda \uparrow \sigma .
$$

The function $R$ is regular in a neighbourhood of the line $\operatorname{Re} \lambda=\sigma$. The zeros of the function $\lambda \mapsto 1-g^{\prime}(0) K(\lambda)$, which lie in a vertical strip left-adjacent to the line $\operatorname{Re} \lambda=\sigma$ lie, in fact, in a rectangle according to the Lemma of Riemann-Lebesgue [16, Section 1.8]. The analyticity of $K$ then implies that there are only finitely many inside this rectangle. Hence, if we choose the width of the strip sufficiently small, then there are no other zeros of $\lambda \mapsto 1-g^{\prime}(0) K(\lambda)$ in this strip, besides the real zero $\lambda=\sigma$, and we can apply Lemma 6.1 (with the obvious modifications) to obtain the statement of the theorem.

The use of an Ikehara-type lemma makes the proof of the asymptotic behaviour rather simple, but has the disadvantage that the conclusion holds only for monotone solutions. Our next objective is to give an alternate proof of the asymptotic behaviour, under slightly different assumptions on $k$, without presupposing that the solution be monotone.

TheOREM 6.3. Suppose $m>0, g^{\prime}(0)>1$, and $g(x) \leqslant g^{\prime}(0) x$ for $x \in[0, p]$. Let $\varepsilon>0$ be such that $g(x)=g^{\prime}(0) x+\mathrm{O}\left(x^{1+\varepsilon}\right)$, cf. $\left(\mathrm{H}_{g}^{2}\right)$. Suppose that $K(-\delta)<\infty$ for some $\delta>0$, and that there exists a $\beta \in(\sigma, \sigma(1+\varepsilon))$ such that (i) $g^{\prime}(0) K(\beta)<1$, and (ii) $k(x) \mathrm{e}^{-[\beta /(1+\varepsilon)] x}$ is bounded. If $u$ is a nontrivial solution of (1.1), then there exists a positive constant $A$ such that $\lim _{x \rightarrow-\infty} u(x) \mathrm{e}^{-\sigma x}=A$.

Proof. In this proof we use for any given function $\phi: \mathbf{R} \rightarrow \mathbf{R}$ the symbol $\phi_{\lambda}$ to denote the mapping $x \mapsto \phi(x) \mathrm{e}^{-\lambda x}, x \in \mathbf{R}$. Let $u$ be a nontrivial solution of (1.1). We know that $u_{\beta /(1+\varepsilon)} \in L_{1}(\mathbf{R})$ and $k_{\beta /(1+\varepsilon)} \in L_{\infty}(\mathbf{R})$. Hence, $u_{\beta /(1+\varepsilon)}(x) \leqslant g^{\prime}(0) u_{\beta /(1+\varepsilon)} * k_{\beta /(1+\varepsilon)}(x) \leqslant C$ or, in other words, $u(x) \leqslant$ $C \mathrm{e}^{-[\beta /(1+\varepsilon)] x}$ for all $x \in \mathbb{R}$. We rewrite (1.1) as an inhomogeneous linear convolution equation,

$$
u(x)=g^{\prime}(0) u * k(x)+r(x) \quad x \in \mathbf{R},
$$

where $r(x):=\left(g \circ u-g^{\prime}(0) u\right) * k(x)$. There exists a positive constant $C$ such that $0 \geqslant r(x) \geqslant$ $-C u^{1+\varepsilon} * k(x) \geqslant-C \mathrm{e}^{-\beta x}$ for all $x \in \mathbf{R}$. Multiplying both sides of (6.1) with $\mathrm{e}^{-\beta x}$ we obtain

$$
u_{\beta}(x)=g^{\prime}(0) u_{\beta} * k_{\beta}(x)+r_{\beta}(x) \quad x \in \mathbf{R} .
$$

The function $r_{\beta}$ is nonpositive and bounded. Consider the iterative scheme $v^{(0)}(x):=r_{\beta}(x)$, $v^{(n)}(x):=g^{\prime}(0) v^{(n-1)} * k_{\beta}(x)+r_{\beta}(x), n \in \mathbf{N}_{+}$. For each $x$, the sequence $\left\{v^{(n)}(x): n \in \mathbf{N}_{+}\right\}$is monotone nonincreasing. Moreover, $v^{(n)}(x) \geqslant \inf \left\{r_{\beta}(x): x \in \mathbf{R}\right\}\left(1+\alpha+\alpha^{2}+\ldots+\alpha^{n}\right)$, where $\alpha=g^{\prime}(0) K(\beta)$ $<1$. So the sequence $\left\{v^{(n)}: n \in \mathbf{N}_{+}\right\}$being bounded and monotone, converges to a limit function, $v$ say, which satisfies the equation $v(x)=g^{\prime}(0) v * k_{\beta}(x)+r_{\beta}(x)$ for all $x \in \mathbf{R}$. The same equation is satisfied by the function $u_{\beta}$, cf. (6.2). Since $v$ is nonpositive and $u_{\beta}$ is positive, the difference $u_{\beta}-v$ is not the zero function. Let the function $w$ be defined by $w_{\beta}(x):=u_{\beta}(x)-v(x)$. Then $w$ satisfies 
the homogeneous linear convolution equation

$$
w(x)=g^{\prime}(0) w * k(x) \quad x \in \mathbf{R},
$$

and the estimates $0 \leqslant w(x) \leqslant C\left(1+\mathrm{e}^{\beta x}\right)$ for all $x \in \mathbf{R}$. We claim that this implies that $w(x)=$ $A \mathrm{e}^{\sigma x}$ for some $A>0$.

In order to support this claim we first collect some information concerning the roots of the characteristic equation $g^{\prime}(0) K(\lambda)=1$ in the strip $S_{\beta}=\{\lambda \in \mathbf{c}: 0 \leqslant \operatorname{Re} \lambda \leqslant \beta\}$. In this strip, $\sigma$ is the only real root; all other roots occur in complex conjugate pairs. The nonnegativity of $k$ implies that the real part of any root other than $\sigma$ is strictly less than $\sigma$. As in the proof of Theorem 6.2 it follows that there are only finitely many roots in $S_{\beta}$.

The function $w$, being a solution of (6.3) which satisfies the estimates $0 \leqslant w(x) \leqslant C\left(1+\mathrm{e}^{\beta x}\right)$, is a linear combination of exponential functions, possibly multiplied by polynomial functions, where the exponents are found as the roots of the characteristic equation in $S_{\beta}$, cf. [16, Section 11.2]. (It is in this step that the assumption $K(-\delta)<\infty$ is used.) Next, observe that any contribution from a pair of complex conjugate roots (with real parts less than $\sigma$ ) leads to an oscillatory behavior of $w$ which is asymptotically dominant over $\mathrm{e}^{\sigma x}$ as $x \rightarrow-\infty$. Hence, if there were a contribution to $w$ from any complex conjugate pair of roots of the characteristic equation in the strip $S_{\beta}, w(x)$ would certainly become negative for some $x$ sufficiently large negative, which is impossible because $w(x)$ is nonnegative, as we have seen. The conclusion is, therefore, that the only nonzero coefficient in the finite expansion for $w$ is the one corresponding to $\sigma$. In other words, $w(x)=A \mathrm{e}^{\sigma x}$ for some $A>0$, as claimed.

Finally, $u(x)=w(x)+v(x) \mathrm{e}^{\beta x}$ and so $\lim _{x \rightarrow-\infty} u(x) \mathrm{e}^{-\sigma x}=\lim _{x \rightarrow-\infty} w(x) \mathrm{e}^{-\sigma x}=A$.

Our final results concern the uniqueness of nontrivial solutions of (1.1). We observe that the homogeneous equation (1.1) is invariant under translation, so uniqueness is to be understood in the sense of uniqueness modulo translation.

THEOREM 6.4. Suppose that the conditions of Theorem 6.3 are satisfied and that, in addition, $g$ is such that $|g(x)-g(y)| \leqslant g^{\prime}(0)|x-y|$ for all pairs $x, y \in[0, p]$. Then there is at most one nontrivial solution (modulo translation) of (1.1).

Proof. Let $u_{1}^{*}$ and $u_{2}$ be two nontrivial solutions of (1.1). There exist positive constants $C^{*}$ and $C$ such that $\lim _{x \rightarrow-\infty} u_{1}^{*}(x) \mathrm{e}^{-\sigma x}=C^{*}$ and $\lim _{x \rightarrow-\infty} u_{2}(x) \mathrm{e}^{-\sigma x}=C$, according to Theorem 6.3. Define $u_{1}$ by putting $u_{1}(x):=u_{1}^{*}(x-\tau)$ for all $x \in \mathbf{R}$, where $\tau=\sigma^{-1} \ln \left(C^{*} / C\right)$. Then $u_{1}$ is also a nontrivial solution of (1.1) and $\lim _{x \rightarrow-\infty} u_{1}(x) \mathrm{e}^{-\sigma x}=C$. Thus, if we consider the function $v$ defined by $v(x):=$ $\left(u_{1}-u_{2}\right)(x) \mathrm{e}^{-\sigma x}$ for all $x \in \mathbf{R}$, then $v$ is continuous on $\mathbf{R}, \lim v(x)=: v( \pm \infty)$ exist, and $v(-\infty)=$ $v(\infty)=0$. Also, if $z:=\sup \{|v(x)|: x \in \mathbf{R}\}$, then $z=v\left(x_{0}\right)$ forsome $x_{0} \in \mathbf{R}$. Now, $v(x)=\left(u_{1}-u_{2}\right)(x) \times$ $\mathrm{e}^{-\sigma x}=\left(g \circ u_{1}-g \circ u_{2}\right) * k(x) \mathrm{e}^{-\sigma x}$, so since the Lipschitz constant of $g$ on $[0, p]$ is at most equal to $g^{\prime}(0)$ we have the inequality $|v(x)| \leqslant g^{\prime}(0)|v| * k_{\sigma}(x)$, where $k_{\sigma}(x):=k(x) \mathrm{e}^{-\sigma x}$, which is valid for all $x \in \mathbf{R}$. From this point on, the arguments parallel some of the arguments used in the proof of Lemma 2.2, cf. also Feller [13, Section XI.2]. Upon induction we obtain the estimate

$$
|v(x)| \leqslant\left(g^{\prime}(0)\right)^{n}|v| * k_{\sigma}^{n *}(x) \quad x \in \mathbf{R},
$$


for any $n \in \mathbf{N}_{+}$. Now, the expression in the right member of this inequality is at most equal to $z\left(g^{\prime}(0)\right)^{n} \int_{\mathbb{R}} k_{\sigma}^{n *}(x) \mathrm{d} x$ which, in turn, is equal to $z\left(g^{\prime}(0) \int_{\mathbb{R}^{\prime}} k_{\sigma}(x) \mathrm{d} x\right)^{n}=z\left(g^{\prime}(0) K(\sigma)\right)^{n}=z$. Hence, at $x=x_{0}$, the inequality (6.4) must be an equality, $\left|v\left(x_{0}\right)\right|=\left(g^{\prime}(0)\right)^{n}|v| * k_{\sigma}^{n^{*}}\left(x_{0}\right)$ for all $n \in \mathbf{N}_{+}$. But this is only possible if $v\left(x_{0}-y\right)=v\left(x_{0}\right)$ for all $y \in \sum$. (We recall that $\sum=\bigcup_{n=1}^{\infty} \sum_{n}, \sum_{n}$ being the set consisting of the points of increase of $k^{n^{*}}$.) It follows that $v(x)=v\left(x_{0}\right)$ for all $\mathrm{x} \in \mathbf{R}$ if $k$ is not concentrated on either $\mathbf{R}_{+}$or $\mathbf{R}_{-}$, cf. Lemma 2.1. If $k$ is concentrated on $\mathbf{R}_{+}$, say, then it follows that $v(x)=v\left(x_{0}\right)$ for all $x \in\left(-\infty, x_{0}-a\right]$ for some $a \in \mathbf{R}_{+}$, but as $v$ satisfies the inequality (6.4) one may conclude again that $v(x)=v\left(x_{0}\right)$ for all $x \in \mathbf{R}$. Hence, $v$ is a constant function on $\mathbf{R}$. Since its limiting values at $\pm \infty$ are zero, it follows that $v$ is the zero function, i.e., $u_{1}(x)=u_{2}(x)$ for all $x \in \mathbb{R}$.

The proof of the following uniqueness theorem is analogous to the proof of Theorem 6.4 .

THEOREM 6.5. Suppose that the conditions of Theorem 6.2 are satisfied and that, in addition, $g$ is such that $|g(x)-g(y)| \leqslant g^{\prime}(0)|x-y|$ for all pairs $x, y \in[0, p]$. Then there is at most one monotone nondecreasing nontrivial solution (modulo translation) of (1.1).

Remark 6.6. If $g$ is monotone nondecreasing and sublinear (i.e., $g(\alpha x) \geqslant \alpha g(x)$ for $\alpha \in[0,1]$, $x \in[0, p])$, then the Lipschitz constant of $g$ on $[0, p]$ is at most $g^{\prime}(0)$. The former properties were used by one of the authors in [17] to prove a uniqueness result. The fact that one can use the less restrictive condition involving the Lipschitz constant was observed by Barbour [8] for a function $g$ which was explicitly given by an expression of the form $g(x)=\alpha\left(1-\mathrm{e}^{-x}\right)$.

Remark 6.7. We observe that a combination of the existence result for monotone solutions (cf. 1,2 ) and the uniqueness result of Theorem 6.4 leads, in a very indirect way, to the conclusion that monotone functions $g$ lead to monotone nontrivial solutions of $(1.1)$ only. It would be of interest to have a direct proof of this fact.

\section{APPLICATION TO A DETERMINISTIC MODEL FROM MATHEMATICAL} EPIDEMIOLOGY

The spatio-temporal development of an epidemic among a closed population, with habitat $\mathbf{R}^{n}$, can be described by an integral equation of the form

$$
u(t, x)=\int_{0}^{\infty} H(\tau) \int_{\mathbb{R}^{n}} g \circ u(t-\tau, y) V(x-y) \mathrm{d} y \mathrm{~d} \tau,
$$

$-\infty<t<\infty, x \in \mathbb{R}^{n}$, cf. [1]. Here $u$ is a measure for the density of susceptible individuals.

The equation is based on various assumptions, the most important being

(i) the members of the population can be categorized as either susceptible to or infected by the disease;

(ii) the infectivity of an infected individual as a function of time elapsed since exposure and position relative to the individual's own position is given by $H: \mathbf{R}_{+} \rightarrow \mathbf{R}$ and $V: \mathbf{R}^{n} \rightarrow \mathbf{R}$, respectively;

(iii) the disease induces permanent immunity, so an individual can pass from the class of susceptibles to the class of infectives, but not vice versa. 
Both $H$ and $V$ are nonnegative, and normalized to have integral one. The assumption that the habitat $\mathbf{R}^{n}$ is homogeneous and isotropic is reflected in the fact that $V$ is a radial function. The nonlinearity of the equation comes about through the function $g$ which has the form $g(x)=$ $\alpha\left(1-\mathrm{e}^{-x}\right)$. The parameter $\alpha$ has a threshold value $1, \mathrm{cf}$. [1,6]; here, we restrict our attention to the more interesting case $\alpha>1$. In the so-called traveling (plane) wave problem one looks for solutions of (7.1) of the form $u(t, x)=w(x \cdot v+c t)$, where $v$ is a fixed unit vector. If one chooses a basis in $\mathbb{R}^{n}$ such that $v=(1,0, \ldots, 0)$, then the function $w$ must satisfy the following convolution equation on the line,

$$
w(x)=(g \circ w) * V_{c}(x) \quad x \in \mathbf{R},
$$

where $\quad V_{c}(x):=\int_{0}^{\infty} H(\tau) \tilde{V}(x-c \tau) \mathrm{d} \tau, \quad x \in \mathbb{R}$, with $\tilde{V}\left(x_{1}\right):=\int_{\mathbb{R}^{n-1}} V\left(x_{1}, x_{2}, \ldots, x_{n}\right) \mathrm{d} x_{2} \ldots \mathrm{d} x_{n}$, $x_{1} \in \mathbf{R}$. If $c=0$ the function $V_{c}$ is symmetric. One can then apply Theorem 4.1, from which it follows that (7.1) does not admit any standing wave solution. Next, we consider the case $c \neq 0$. Because of the symmetry of $V$ we can restrict our attention to the case $c>0$.

As $c$ increases, the mass of $V_{c}$ shifts to the right. This can be seen by inspecting the qualitative behavior of the characteristic function $L_{c}$, associated with the linearized equation, as a function of the wave velocity $c$. By definition,

$$
\begin{aligned}
L_{c}(\lambda) & :=g^{\prime}(0) \int_{\mathbf{R}} \mathrm{e}^{-\lambda y} V_{c}(y) \mathrm{d} y \\
& =g^{\prime}(0) \int_{\mathbf{R}_{+}} \mathrm{e}^{-\lambda c \tau} H(\tau) \mathrm{d} \tau \int_{\mathbf{R}} \mathrm{e}^{-\lambda y} \tilde{V}(y) \mathrm{d} y .
\end{aligned}
$$

The nonnegativity of $H$ and $V$ implies that, for $c$ fixed, $L_{c}$ is a convex function of $\lambda(\lambda \in \mathbf{R})$ and, for $\lambda$ fixed $(\lambda>0)$, it is a monotonically decreasing function of $c$. Furthermore, $L_{c}(0)=g^{\prime}(0)=\alpha>1$. Hence, the number $c_{0}:=\inf \left\{c>0\right.$ : there exists $\lambda>0$ such that $\left.L_{c}(\lambda)=1\right\}$ is well-defined if $H$ and $V$ satisfy the appropriate hypotheses [1].

Various authors have given constructive proofs for the existence of (monotone) nontrivial solution of (7.2) in the case $c \geqslant c_{0}, \mathrm{cf}$. $[1,2,7,9]$. From the results of our investigation it follows that there exists exactly one such solution (modulo translation) at each speed $c>c_{0}$, at least if $\tilde{V}_{\lambda}: x \mapsto \tilde{V}(x) \mathrm{e}^{-\lambda x}$ is bounded for the value of $\lambda$ specified in Theorem 6.4. (If, for instance, $V$ has compact support then this condition is certainly fulfilled.) A similar conclusion has been reached at by Barbour [8] who used probabilistic arguments.

Furthermore, it follows from the results of our investigation that no traveling waves exist with speed $c<c_{0}$. The same result has been obtained for the special case $H(t)=a \mathrm{e}^{-\mu t}(a, \mu$ nonnegative constants) by Atkinson and Reuter [7] and by Aronson [6], and for a discrete time model in population genetics by Weinberger [2]. Both Aronson and Weinberger based their proofs on the construction of a subsolution. This approach made it actually possible for them to conclude that $c_{0}$ is the asymptotic speed of propagation of disturbances from a rest state of the associated initial value problem (see also Mollison [12]).

In our opinion, the main advantages of the approach presented in this paper is that the same chain of purely analytical arguments leads to the nonexistence, as well as the uniqueness results.

Acknowledgement. Part of this investigation was carried out while the second author was on foreign assignment to the Mathematisch Centrum, Amsterdam (Neth.). The final draft of this paper was written while the first author was visiting the Applied Mathematics Division at Argonne National Laboratory. Both authors wish to thank their respective host institutions for their hospitality and financial support. 


\section{REFERENCES}

1. Diekmann O., Thresholds and travelling waves for the geographical spread of infection, Mathematical Centre Report TW 166, Amsterdam (1977).

2. WEINBERGER H. F., Asymptotic behaviour of a model in population genetics, to appear in: Indiana University Seminar in Applied Mathematics, J. Chadam, ed., Springer Lecture Notes.

3. Karlin S., On the renewal equation, Pacif. J. Math. 5, 229-257 (1955)

4. Essén M., Studies on a convolution inequality, Ark. Mat. 5, 113-152 (1963).

5. Rudin W., Functional Analysis. McGraw-Hill, New York (1973).

6. Aronson D. G., The asymptotic speed of propagation of a simple epidemic, in: Nonlinear Diffusion, W. E. Fitzgibbon \& H. F. Walker, eds., Research Notes in Mathematics, Pitman Publishing Co. (1977).

7. Atkinson C. \& Reuter G. E. H. Deterministic epidemic waves, Math. Proc. Camb. phil. Soc. 80, 315-330 (1976)

8. Barbour A. D., The uniqueness of Atkinson and Reuter's epidemic waves, Math. Proc. Camb. phil. Soc. 82, 127-130 (1977).

9. Brown K. J. \& Carr J., Deterministic epidemic waves of critical velocity, Math. Proc. Camb. phil. Soc. 81, 431-435 (1977).

10. Kendall D. G., Mathematical models of the spread of infection, in: Mathematics and Computer Science in Biology and Medicine, pp. 213-224. Medical Research Council, London (1965).

11. Kermack W. O. \& McKendrick A. G., A contribution to the mathematical theory of epidemics, Proc. R. Soc. A $115,700-721$ (1927).

12. Mollison D., The rate of spatial propagation of simple epidemics, in: Proc. Sixth Berkeley Symposium on Mathematical Statistics and Probability, Vol. III, pp. 579-614. L. M. le Cam, J. Neyman \& E. L. Scott, eds. Univ. of California Press, Berkeley (1972).

13. Feller W., An Introduction to Probability Theory and Its Applications, Vol. II. Wiley, New York (1966).

14. Rudin W., Fourier Analysis on Groups. Interscience, New York (1962).

15. WIDDER D. V., The Laplace Transform. University Press, Princeton (1946).

16. Trtchmarsh E. C., Introduction to the Theory of Fourier Integrals. Clarendon Press, Oxford (1937).

17. DiekmanN O., Limiting behaviour in an epidemic model, Nonlinear Analysis, Theory, Methods Applic. 1, 459-470 (1977) 